\title{
Buffer Layers, Defects, and the Capacitance Step in the Admittance Spectrum of a Thin-Film Solar Cell
}

\author{
Florian Werner ${ }^{*}$ and Susanne Siebentritt \\ Laboratory for Photovoltaics, Physics and Materials Science Research Unit, \\ University of Luxembourg, Rue du Brill 41, L-4422 Belvaux, Luxembourg
}

\section{(Received 10 November 2017; revised manuscript received 1 March 2018; published 30 May 2018)}

\begin{abstract}
Defects in complex multilayered thin-film solar cells are often analyzed by capacitance-based techniques, which originally were developed for simple homojunctions in single-crystal bulk semiconductors. We discuss the impact of a capacitive and conductive buffer layer on the impedance and capacitance spectra of thin-film solar cells. While the resulting capacitance spectra are indistinguishable from those caused by a deep defect level, the buffer layer and $p$ - $n$ junction can clearly be distinguished in the experimental impedance spectra. Exploiting bias voltage and illumination as additional experimental parameters allows us to test a given hypothesis-deep defect or buffer layer-to explain characteristic capacitance steps in thermal admittance spectroscopy of thin-film solar cells. We address the controversial origin of the $N 1$ signature commonly observed in admittance spectroscopy of $\mathrm{Cu}(\mathrm{In}, \mathrm{Ga}) \mathrm{Se}_{2}$ solar cells. The circuit element dominantly defining the main capacitance step in our devices is unaffected by applied bias voltage, and its conductivity increases linearly with illumination intensity. We conclude that the main capacitance step in our devices is most plausibly explained by the presence of a buffer layer connected in series to the $p-n$ junction of the device and is not related to any deep defects or mobility freeze-out.
\end{abstract}

DOI: 10.1103/PhysRevApplied.9.054047

\section{INTRODUCTION}

Measurements of the temperature- and frequencydependent capacitance (thermal admittance spectroscopy) are among the most important device characterization techniques to study defects in solar-cell devices [1]. In principle, all relevant recombination parameters of a defect can be deduced by studying capacitance steps arising due to its finite response time [1-5]. Such capacitance steps are indeed routinely observed in the admittance spectra of thin-film solar cells of a variety of absorber materials, for example, in chalcopyrite $\mathrm{Cu}(\mathrm{In}, \mathrm{Ga}) \mathrm{Se}_{2}$ (CIGS, discussed in more detail below), kesterite $\mathrm{Cu}_{2} \mathrm{ZnSnSe}_{4}[5,6]$, and $\mathrm{CdTe}[7,8]$, and extensive characterization of the corresponding alleged defects has been collected over many years.

In the case of CIGS solar cells, one particular admittance signature known as the " $N 1$ level" [9] has been observed universally in many devices (see, e.g., Ref. [10]) and has been discussed controversially for two decades already. Primarily, it has been attributed to a defect, although

\footnotetext{
*florian.werner@uni.lu
}

Published by the American Physical Society under the terms of the Creative Commons Attribution 4.0 International license. Further distribution of this work must maintain attribution to the author(s) and the published article's title, journal citation, and DOI. conflicting evidence exists concerning where these defects are located within the device $[9,11,12]$. This standard interpretation was recently challenged by an increasing number of publications which suggest alternative explanations for the $N 1$ level, mostly linked to the transport characteristics of the solar-cell absorber $[13,14]$ or to a transport barrier [7,15-21] within the device.

This controversy concerning the interpretation of capacitance steps in admittance spectroscopy of thin-film solar cells is related to the complex multilayered device architecture, which is sketched in Fig. 1 for a typical CIGSbased solar cell. Most standard models for defect characterization were originally developed for single-crystal bulk or wafer-based semiconductor devices with comparably simple device geometries. All parts of these devices located outside of the space-charge region in the active absorber can usually be treated as a pure resistor (e.g., the series resistance of the quasineutral bulk and contacts) or as a pure capacitance (e.g., the dielectric oxide layer in metal-oxidesemiconductor devices) [2]. This is no longer true for thinfilm devices, where additional layers on top of the active absorber must be conductive to a certain extent but might also affect the total device capacitance. In a recent paper [21], we demonstrated that the CdS buffer layer in a typical $\mathrm{CdS} / i-\mathrm{ZnO} / \mathrm{ZnO}: \mathrm{Al}$ buffer and window stack might indeed introduce a resistive and capacitive transport barrier in the finished device, and its impact on the capacitance spectrum must, thus, be considered carefully. We proposed 


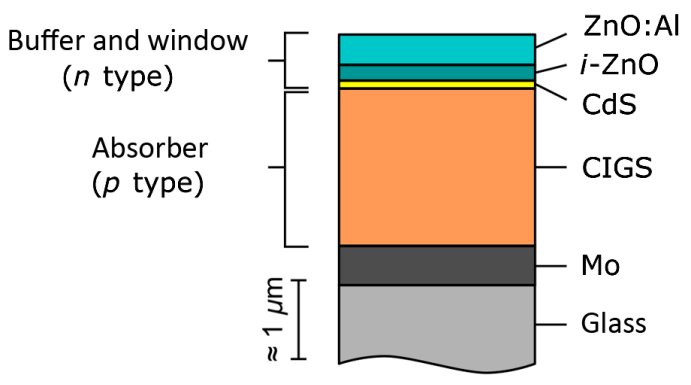

FIG. 1. Sketch of a typical CIGS thin-film solar cell processed on a glass substrate: Mo back contact, $p$-doped CIGS absorber layer, CdS buffer layer, and $n$-type window double layer (nonintentionally-doped "intrinsic" $i$ - $\mathrm{ZnO}$ and $\mathrm{Al}$-doped $\mathrm{ZnO}$ ). The given scale is only approximate.

that this CdS buffer layer is a likely candidate to explain the universality of the $N 1$ signature for CIGS solar cells.

Conventional electrical characterization of solar cells focuses on the admittance or capacitance spectrum since capacitive contributions of deep defects and the spacecharge region are additive [1]. Because of the series connection of the junction diode and buffer layer in the electrical equivalent circuit, however, characterization of thin-film devices certainly benefits from taking into account the complex impedance spectrum as well. In this way, the components of the device contributing individually to the total frequency response can be identified and separated. A more comprehensive model of the device can be established further by making use of the bias and illumination dependence of the device impedance in addition to the frequency and temperature dependence studied in standard thermal admittance spectroscopy. Note that quantitative electrical characterization of polycrystalline thin-film solar cells compared to single-crystal silicon devices might require even more modifications [22] beyond the consideration of additional layers, but these modifications are not addressed here since we are concerned about the fundamental interpretation of the data rather than the quantitative details.

Impedance spectroscopy has also been applied previously to thin-film solar cells both for CIGS [14,23-25] and CdTe $[8,26,27]$ devices. These studies, however, mostly focused on damage and inhomogeneities around the CdS/ CIGS interface in CIGS devices and a complete device model comprising several defect levels and a back contact barrier in CdTe devices. Although several of these earlier studies mention buffer layers at the front of the device in passing, a more specific treatment of the ubiquitous buffer layer and its impact on capacitance spectra in standard thermal admittance spectroscopy is certainly warranted.

In this manuscript, we discuss the relations between the impedance and capacitance spectrum for a thin-film solar cell containing a $p-n$ junction and a capacitive but conductive buffer layer. We demonstrate that a characteristic frequency dependence of the capacitance identical to that of a deep defect must be expected naturally for thin-film solar cells, even in the absence of any deep defects, simply due to the presence of a buffer layer in the device architecture. In Sec. II, we present the fundamental equations describing the admittance of a $p-n$ junction in series with a buffer layer and discuss the similarities to the frequency response of a defect level. In Sec. III, we focus on the origin of the controversial $N 1$ level in CIGS solar cells and show how the impact of a buffer layer and deep defects can be distinguished in the impedance spectrum even if the resulting capacitance spectra are identical for both scenarios. An analysis of the voltage- and illumination-dependent impedance spectra provides direct experimental evidence that the main capacitance step observed in the admittance spectroscopy of our devices is indeed caused by the presence of a buffer layer rather than deep defects.

Although the present manuscript focuses on CIGS solar cells with a CdS buffer layer, we expect that our results also apply to other thin-film technologies and different material combinations due to the fundamentally similar device architecture.

\section{FREQUENCY-DEPENDENT CAPACITANCE}

\section{A. Serial electrical equivalent circuit}

Experimental admittance spectra of single-junction solar cells are typically analyzed based on the parallel equivalent circuit model shown in Fig. 2(a), which consists of a single parallel capacitance $C_{p}$ and a parallel conductance $G_{p}$. For an ideal solar cell without any deep defects, $G_{p}$ and $C_{p}$ are independent of ac frequency, $G_{p}$ is given by the voltage derivative of the exponential diode current plus any shunt conductance bypassing the diode, and $C_{p}$ is equal to the space-charge region capacitance of the $p-n$ junction. In experiment, however, both conductance $G_{p}$ and capacitance $C_{p}$ are typically found to vary with ac frequency. Although this observation is conventionally explained by a frequency-dependent response of deep defects within the device [3], the mere presence of a buffer layer naturally

(a)
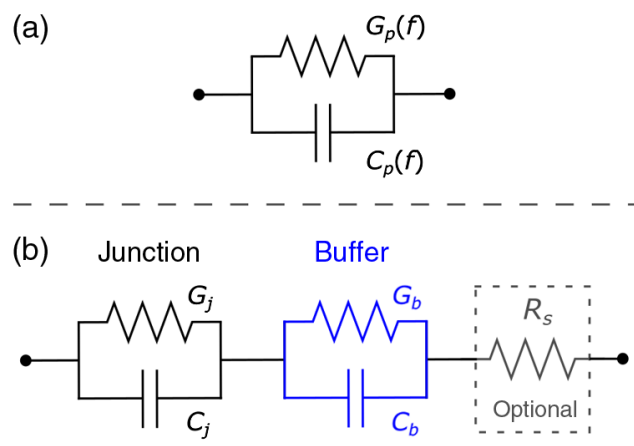

FIG. 2. Electrical equivalent circuits: (a) parallel model used in admittance spectroscopy and (b) series connection of the junction and buffer elements, including an optional lumped series resistance. 
results in an identical frequency response as we discuss below.

The presence of a buffer layer in a thin-film solar cell adds an additional resistive and capacitive contribution to the small-signal admittance of the complete device, which is not included in the simple parallel model shown in Fig. 2(a). The buffer layer, thus, represents an additional circuit element in series with the junction element. The most fundamental electrical equivalent circuit model for a typical thin-film solar cell then consists of the series connection of two $G$ - $C$ elements (also referred to as $R C$ elements, with $R=1 / G$ ), as sketched in Fig. 2(b). One of these elements represents the buffer layer $\left(G_{b}\right.$ and $\left.C_{b}\right)$, the other element originates from the space-charge region of the $p-n$ junction $\left(G_{j}\right.$ and $\left.C_{j}\right)$. In order to correctly describe the admittance spectrum at high frequencies of a few $100 \mathrm{kHz}$ and above, it is often necessary to additionally consider a lumped series resistance $R_{s}$, which accounts for Ohmic losses in the external contacts, transparent conductive oxide layer, and the quasineutral region of the absorber. Ohmic series resistance effects, however, are inconsequential for our discussion below and are mostly neglected.

\section{B. Impedance and capacitance spectra}

Because of the series connection of two $G$ - $C$ elements, it is helpful to look at the impedance $Z$ rather than the admittance $Y$. The admittance of a single $G$ - $C$ element is $Y=G+i \omega C$, which is inverted to yield the impedance

$$
Z=\frac{1}{Y}=\frac{1}{G+i \omega C}=\frac{G-i \omega C}{G^{2}+(\omega C)^{2}}=\frac{1}{G} \frac{1-i \omega \tau}{1+(\omega \tau)^{2}}
$$

with characteristic time constant $\tau=C / G$. Equation (1) describes a semicircle in the complex $Z$ plane, and the real and imaginary parts of the impedance show a step and peak, respectively, at a characteristic angular inflection frequency $\omega_{\text {char }}$ equal to the inverse time constant $\omega_{\text {char }}=1 / \tau$. Note that this frequency dependence naturally occurs due to the arithmetic in inverting the complex admittance, although both conductance $G$ and capacitance $C$ themselves are assumed to be independent of frequency. Further note the minus sign in the numerator on the right-hand side of Eq. (1), indicating that the imaginary part of the impedance is, in fact, negative for a capacitive circuit. To simplify log scaling of the data, we often implicitly ignore this minus sign in the following.

In order to study the impact of the buffer layer on the capacitance spectrum in thermal admittance spectroscopy, the respective impedances according to Eq. (1) of all individual $G$-C elements in Fig. 2(b) are added to yield the total device impedance. The effective conductance $G_{\text {eff }}$ and capacitance $C_{\text {eff }}$ for such an electrical equivalent circuit are then obtained from the real and imaginary parts, respectively, of the total admittance, which requires a further inversion of the total device impedance:

$$
G_{\text {eff }}+i \omega C_{\text {eff }}=\frac{1}{Z_{\text {sum }}}=\frac{1}{\sum_{k} Z_{k}(\omega)} .
$$

By performing these calculations for two frequencyindependent $G-C$ elements, in our case, for the buffer layer $\left(Y_{b}=G_{b}+i \omega C_{b}\right)$ and the solar-cell junction $\left(Y_{j}=\right.$ $G_{j}+i \omega C_{j}$ ), we obtain a capacitance step of the effective capacitance according to

$$
C_{\mathrm{eff}}(\omega)=C_{\infty}+\frac{\Delta C}{1+\left(\omega / \omega_{t}\right)^{2}},
$$

where $1 / C_{\infty}=1 / C_{j}+1 / C_{b}$ is the high-frequency limit of the effective capacitance, $\Delta C=\alpha\left(C_{j}+C_{b}\right)$ is the capacitance step height with $0<\alpha<1$ depending on both $G$ and $C$ values [compare Eq. (5)], and the inflection frequency $f_{t}$ is given by

$$
f_{t}=\frac{\omega_{t}}{2 \pi}=\frac{1}{2 \pi} \frac{G_{b}+G_{j}}{C_{b}+C_{j}} .
$$

Note that the capacitance spectrum described by Eqs. (3) and (4) represents a capacitance step at a finite inflection frequency $f_{t}$, although all individual elements of the equivalent circuit in our calculation are by definition independent of the frequency.

The parameter $\alpha$ in Eq. (1) is a scaling factor given by

$$
\alpha=\left[\frac{R_{b}}{R_{b}+R_{j}} \frac{C_{b}}{C_{b}+C_{j}}-\frac{R_{j}}{R_{b}+R_{j}} \frac{C_{j}}{C_{b}+C_{j}}\right]^{2}
$$

with $R_{b}=1 / G_{b}$ and $R_{j}=1 / G_{j}$. For most reasonable solar cells at relevant temperatures, we can assume the buffer layer to be significantly more conductive than the junction, i.e., $R_{b} \ll R_{j}$ by several orders of magnitude. If we further assume that the buffer and junction capacitances are not orders of magnitude different, Eq. (5) simplifies to

$$
\alpha \approx\left[\frac{C_{j}}{C_{b}+C_{j}}\right]^{2} .
$$

Applying the same approximation, the low-frequency limit of the effective capacitance according to Eq. (3) approaches the junction capacitance $C_{\text {eff }}(\omega=0)=$ $C_{\infty}+\Delta C \approx C_{j}$. Note that the low-frequency capacitance approaching the space-charge region capacitance has farreaching implications beyond admittance spectroscopy, in particular, on the estimation of dopant concentrations based on capacitance-voltage $(C-V)$ measurements $[1,28]$ : since capacitance steps are typically ascribed to defects, the dopant concentration is estimated from the high-frequency limit of the capacitance to reduce the impact of such defects. In contrast, if a buffer layer is responsible for this capacitance step, the space-charge region capacitance-and, 
consequently, the dopant concentration as well-should be derived from the low-frequency limit of the capacitance.

Further refinement of the equivalent circuit might be necessary for realistic devices, for example, by including a lumped series resistance $R_{s}$ as shown in the gray part of Fig. 2(b). For moderate series resistance $\left(R_{s} G_{\text {eff }} \ll 1\right)$, the capacitance spectrum $C_{\text {eff }}^{*}(\omega)$ including the series resistance can be approximated by

$$
C_{\mathrm{eff}}^{*}(\omega)=\frac{C_{\mathrm{eff}}(\omega)}{1+\left(\omega R_{s} C_{\mathrm{eff}}\right)^{2}},
$$

where $C_{\text {eff }}$ is calculated according to Eq. (3). Ohmic series resistance, thus, gives rise to an additional capacitance step, typically at high frequencies above a few $100 \mathrm{kHz}$, with vanishing capacitance in the high-frequency limit.

\section{Temperature dependence of the inflection frequency}

A key signature of an experimental capacitance spectrum is not only the occurrence of the capacitance steps themselves but also that their respective inflection frequencies obey a specific temperature dependence. For the series connection of the $G-C$ elements representing the buffer layer and junction, the inflection frequency of the corresponding capacitance step is given by Eq. (4). Its temperature dependence is, thus, solely determined by the temperature dependence of the conductances and capacitances of the junction and buffer layer. As we argue in the following paragraphs, the temperature dependence of the inflection frequency is, in fact, mostly determined by the conductivity of the buffer layer.

It is often found in experiment that the low- and highfrequency plateaus around a capacitance step do not vary much with the temperature. This can also be observed in the exemplary capacitance spectra shown in Fig. 3 (experimental details are given in Sec. III B), where the plateaus around the main capacitance step are located approximately at $C=10$ and $15 \mathrm{nF} / \mathrm{cm}^{2}$ independent of temperature.

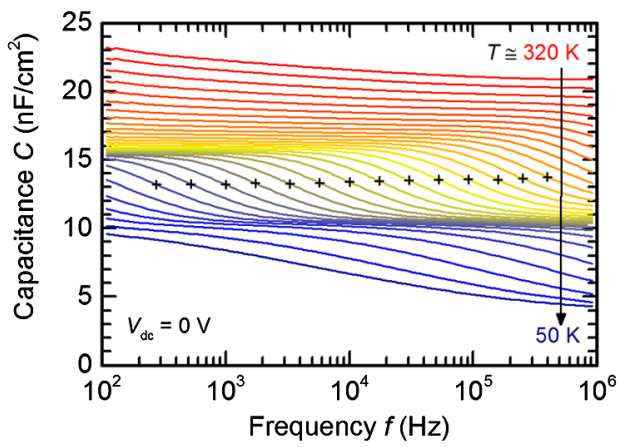

FIG. 3. Exemplary temperature-dependent capacitance spectra of a typical $\mathrm{Cu}$-poor $\mathrm{Cu}(\mathrm{In}, \mathrm{Ga}) \mathrm{Se}_{2}$ thin-film solar cell in a temperature range of $T \approx 320-50 \mathrm{~K}$. Inflection frequencies of the main capacitance step are indicated by black crosses.
We, thus, expect the individual capacitance values $C_{j}$ and $C_{b}$ of the junction and buffer layer to be fairly constant in the relevant temperature range. Even in cases where this assumption might be unjustified, it is unlikely that the capacitance changes over orders of magnitude. Note that the second capacitance step with capacitance values below $10 \mathrm{nF} / \mathrm{cm}^{2}$ apparent from Fig. 3 at low temperatures is attributed to a conductivity freeze-out of the absorber and is not discussed here further.

If both capacitance values are nearly constant with the temperature, any significant temperature dependence of the inflection frequency due to a transport barrier must be attributed to the temperature dependence of the conductances $G_{j}$ and $G_{b}$ of the junction and barrier. Without applying a significant forward bias voltage, the conductance of the $p-n$ junction diode in the dark will be negligible compared to the conductance through the buffer and window stack for any reasonably good solar cell in a wide temperature range. Accordingly, the temperature dependence of the inflection frequency is dominated by the conductance of the buffer layer:

$$
f_{t}(T)=\frac{1}{2 \pi} \frac{G_{b}+G_{j}}{C_{b}+C_{j}} \approx \text { const } \times G_{b}(T) .
$$

In particular, for the simple model of a thermally activated buffer conductance $G_{b}=G_{b .0} \exp \left(-E_{a} / k T\right)$, the inflection frequency of the capacitance step is then approximately

$$
f_{t}(T) \approx \frac{1}{2 \pi} \frac{G_{b .0}}{C_{b}+C_{j}} \exp \left(-\frac{E_{a}}{k T}\right) .
$$

\section{Inflection frequencies of a defect level}

In our serial model that we present above, Eq. (3) predicts a capacitance step due to the presence of the $G-C$ series element representing the buffer layer. The functional form of Eq. (3) at a given temperature is identical to the capacitance response expected for a deep defect within the space-charge region or at the buffer-absorber interface, although the meaning of the parameters in Eq. (3) are different for a defect signature: $C_{\infty}$ is identified with the depletion capacitance of the space-charge region, and $\Delta C$ is proportional to the defect concentration [1]. Accordingly, the presence of a buffer layer in series with the spacecharge region results in an admittance spectrum which can easily be mistaken as a signature of deep defects.

If a given capacitance step is caused by a defect, the energetic depth of the defect level can be inferred from the activation energy of the inflection frequency. The simplest form of a thermally activated inflection frequency with activation energy $E_{a}$ is given by

$$
f_{t}=X_{0} \exp \left(-\frac{E_{a}}{k T}\right),
$$


where ideally, $X_{0}$ is a constant. In a practical experiment, however, the preexponential factor $X_{0}$ in the Arrhenius law often shows a weak temperature dependence itself. For a hypothetical "ideal" defect, $X_{0}$ is expected to scale with $T^{2}$ in first approximation due to the weak temperature dependence of thermal velocity $v_{\text {th }}$ and the effective density of states $N_{C, V}$ in the conduction or valence band of the $n$ - or $p$-type semiconductor. In this case, the inflection frequency is given by the equation [1]

$f_{t}=\frac{1}{2 \pi} v_{\text {th }} N_{C, V} \sigma_{n, p} \exp \left(-\frac{E_{a}}{k T}\right)=\frac{1}{\pi} \xi_{0} T^{2} \exp \left(-\frac{E_{a}}{k T}\right)$,

where $\sigma_{n, p}$ is the electron or hole capture cross section of the defect, and the prefactor $\xi_{0}$ is now assumed to be independent of the temperature. Accordingly, the activation energy $E_{a}$ is typically obtained from an Arrhenius plot of $\ln \left(f_{t} T^{-2}\right)$ vs $1 / T$ rather than $\ln \left(f_{t}\right)$ vs $1 / T$. Whether such a temperature scaling is justified is not known a priori without establishing the exact origin of the capacitance step, and we report it here only for comparison to published literature data, where such a scaling is widely used.

Comparing Eqs. (9) and (11), we find that the impact of a buffer layer on experimental admittance spectra can indeed be easily misinterpreted as a deep defect, as long as the conductance through the buffer layer at least approximately resembles a thermally activated behavior. Previously, we have shown [21] that the conductance of the CdS buffer layer commonly employed in thin-film solar cells might indeed show a thermally activated behavior. In addition, experimentally determined inflection frequencies commonly show deviations from the ideal thermally activated behavior described by Eq. (11). Figure 4 shows the inflection frequencies derived for the main capacitance step in Fig. 3 both without scaling (black, left) and scaled by $T^{-2}$ (red, right) to account for a temperature-dependent

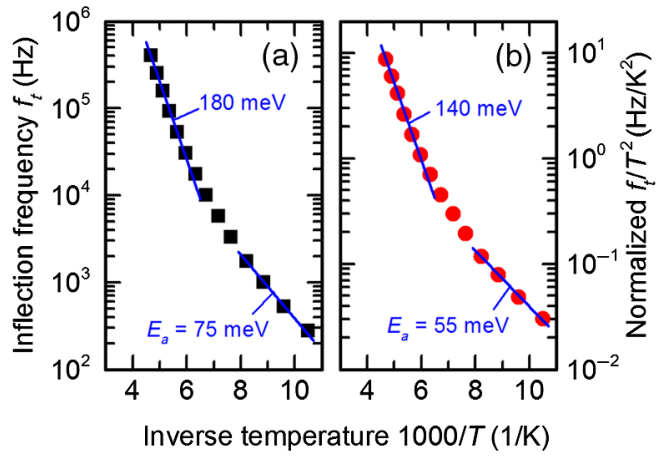

FIG. 4. Inflection frequencies $f_{t}$ of the main capacitance step in Fig. 3 as a function of inverse temperature 1000/T: (a) without scaling and (b) scaled by $T^{-2}$ according to the ideal defect model. Blue lines are exponential fits to obtain the apparent activation energy $E_{a}$ in the high- and low-temperature range. preexponential factor in the Arrhenius law for a simple defect model; see Eq. (11). It is evident that the inflection frequencies do not lie on a straight line in the Arrhenius plots either with or without the $T^{-2}$ scaling. Accordingly, the apparent activation energy $E_{a}$ of the assumed defect level varies between 55 and $180 \mathrm{meV}$ in our example, depending on the temperature range and scaling used in the analysis. Although this deviation can be attributed to temperature-dependent capture cross sections of a defect, different transport processes through the buffer layer provide a plausible and straightforward alternative explanation for the nonideal temperature dependence of the experimental inflection frequencies.

\section{EXPERIMENTAL IMPEDANCE SPECTRA}

\section{A. Deconvolution of experimental impedance spectra}

Based on the discussions in Sec. II B, we find that each $G-C$ element in the electrical equivalent circuit adds a step or peak at its characteristic frequency to the real or imaginary part of the total device impedance. By finding these characteristic frequencies and the corresponding step or peak heights in an experimental impedance spectrum, all relevant parameters (here, the individual conductances and capacitances of the buffer layer and junction) can, in principle, be reconstructed. In practice, an accurate reconstruction is often difficult. Because of the limited frequency range accessible in experiment, different peaks can be resolved only if they are fairly close to each other, in which case, the important features might be obscured by overlap. Such overlap of different peaks is particularly problematic for the case of thin-film solar cells, since the peak or step height scales with resistance, which likely differs by several orders of magnitude between the junction and buffer layer. The experimental impedance spectra shown in Fig. 5 and discussed in the following section clearly illustrate the challenge: The second feature around $f \approx 70 \mathrm{kHz}$ is barely noticeable in the imaginary part of the impedance, and in reverse bias, it starts to be obscured by the low-frequency feature also in the real part of the impedance. This problem can be partly mitigated by plotting the real part of the impedance multiplied by the angular frequency, $\operatorname{Re}(\omega Z)$, rather than the impedance. For a single $G-C$ circuit element, this normalized impedance is given by

$$
\operatorname{Re}(\omega Z)=\frac{1}{C} \frac{\omega \tau}{1+(\omega \tau)^{2}},
$$

again with characteristic time constant $\tau=C / G$, which scales with the inverse capacitance and is, thus, often helpful in separating the signatures of the junction and buffer layer. Furthermore, the characteristic frequencies of the junction and buffer circuit elements might shift significantly with the temperature or applied dc bias voltage, for example, due to reduced conductivity at low temperatures or due to the bias dependence of the space-charge region capacitance. 


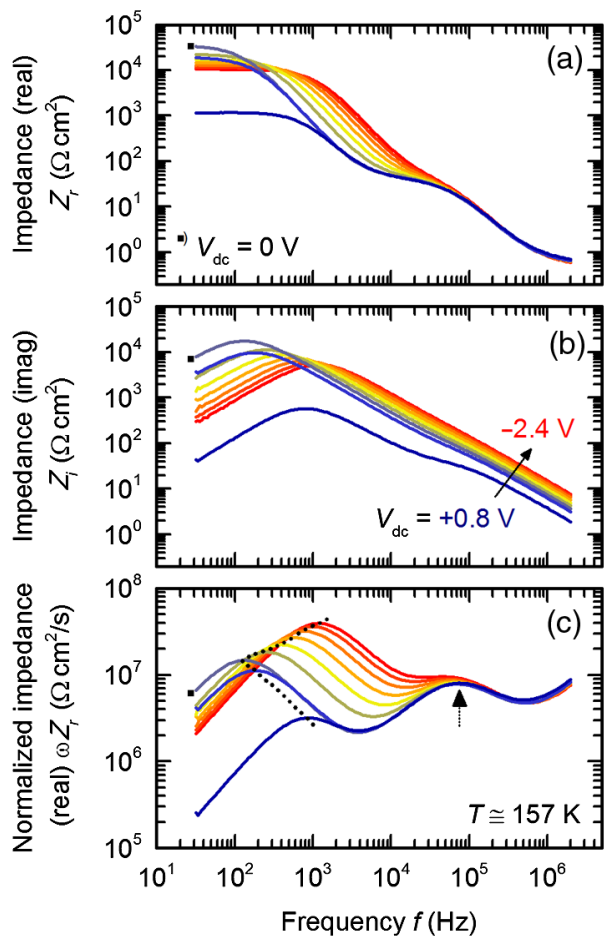

FIG. 5. Impedance spectra of a $\mathrm{Cu}(\mathrm{In}, \mathrm{Ga}) \mathrm{Se}_{2}$ thin-film solar cell with CdS buffer layer at a sample temperature of $T \approx 157 \mathrm{~K}$ and dc bias voltages in the range of $+0.8 \mathrm{~V}$ (blue) to $-2.4 \mathrm{~V}$ (red) in steps of $0.4 \mathrm{~V}$ : (a) real part $Z_{r}$ and (b) imaginary part $Z_{i}$ of the device impedance and (c) real part of the normalized impedance $\omega Z_{r}$. Zero-bias spectra are marked with a black square. Characteristic frequencies of two $G$ - $C$ elements are indicated in (c) by the black dotted line and black arrow.

Confidence in the results of the analysis can, thus, be greatly improved if the impedance spectra are measured over a diverse parameter space.

We combine two different approaches to obtain the individual conductance and capacitance values of each circuit element from experimental impedance spectra.

(i) Peak analysis: According to Eqs. (1) and (12), the inverse conductance and capacitance values of a circuit element are determined by twice the peak height of $Z_{i}(\omega)$ and $\omega Z_{r}(\omega)$, respectively. In the first approximation, we ignore any overlap between neighboring peaks. For a peak with characteristic angular frequency $\omega_{t}$, we, thus, define $G=\left[2 Z_{i}\left(\omega_{t}\right)\right]^{-1}$ and $C=\left[2 \omega_{t} Z_{r}\left(\omega_{t}\right)\right]^{-1}$. Note that the independently determined values of $G$ and $C$ must obey $\omega_{t}=G / C$ for consistent results, and obviously the characteristic frequencies of $Z_{i}(\omega)$ and $\omega Z_{r}(\omega)$ must be identical.

(ii) Numerical regression: We model the total device impedance based on Eq. (1) and obtain all individual conductance and capacitance values from a least-squares regression to the experimental data. In some cases, a constant phase element (CPE) rather than an ideal $G-C$ element is required to accurately describe the impedance spectra of thin-film solar cells [24-26].
Both approaches usually yield very similar results (see Appendix A), unless overlap between peaks or nonidealities resulting in CPE behavior have to be taken into account. In the case of overlapping peaks, we refer to the regression results, and in the case of CPE behavioralbeit minor in this study-we refer to the peak results.

\section{B. Bias dependence of the experimental impedance spectra}

As a practical example, we study the admittance spectra of a typical $\mathrm{Cu}$-poor CIGS thin-film solar cell as a function of the temperature, bias voltage, and illumination intensity. The polycrystalline CIGS absorber is grown on Mo-coated sodalime glass in a three-stage coevaporation process and has a copper content of $[\mathrm{Cu}] /([\mathrm{Ga}]+[\mathrm{In}]) \approx 0.99$ and gallium content of $[\mathrm{Ga}] /([\mathrm{Ga}]+[\mathrm{In}]) \approx 0.37$ as determined from energy-dispersive $\mathrm{x}$-ray measurements. The front stack of the solar cell consists of a CdS buffer layer deposited by chemical bath deposition, a rf-sputtered $i-\mathrm{ZnO} / \mathrm{ZnO}: \mathrm{Al}$ double window layer and a $\mathrm{Ni} / \mathrm{Al}$ front contact grid defined by electron-beam evaporation through a shadow mask. Solar cells with an active area of $0.2-0.5 \mathrm{~cm}^{2}$ are defined by mechanical scribing and achieve efficiencies above $16 \%$ at room temperature under 1-sun illumination. For impedance characterization, the samples are mounted in the dark in a closed-cycle cryostat at a base pressure below $10^{-3}$ mbar. The admittance spectrum is recorded in a frequency range of $f=30 \mathrm{~Hz}-2 \mathrm{MHz}$ with ac voltage amplitude of $30 \mathrm{mV}$ rms. A standard parallel equivalent circuit model, as shown in Fig. 2(a), is used to separate the effective values of conductance $G$ and capacitance $C$. A temperature sensor glued onto an identical glass substrate beside the solar cell is used to estimate the actual temperature of the solar cell, which typically deviates from the set temperature due to the low thermal conductivity of the glass substrate.

Experimental capacitance spectra in a measured temperature range of approximately 320-50 K (set temperature $320-20 \mathrm{~K}$ ) at zero applied dc bias are shown in Fig. 3. For a detailed circuit analysis, Fig. 5 shows the real part $Z_{r}$ and imaginary part $Z_{i}$ of the impedance, as well as the real part $Z_{r}$ multiplied by angular frequency $\omega$, or normalized impedance, measured at a sample temperature of $T \approx$ $157 \mathrm{~K}$ (set temperature $T=150 \mathrm{~K}$ ) for dc bias voltages in a range of -2.4 to $+0.8 \mathrm{~V}$ (some graphs omitted for clarity). Since all features in the impedance spectra shift with the temperature, the particular temperature for each graph in this paper, e.g., $T \approx 157 \mathrm{~K}$ in Fig. 5, is chosen to most clearly depict all relevant impedance features within the experimental frequency range.

From the impedance spectra in Fig. 5, we can clearly identify two distinct features with their respective characteristic frequencies visualized by the black arrow and dotted line in Fig. 5(c): one feature is visible in the lowfrequency range below a few kilohertz and changes significantly with applied bias voltage, and the second 

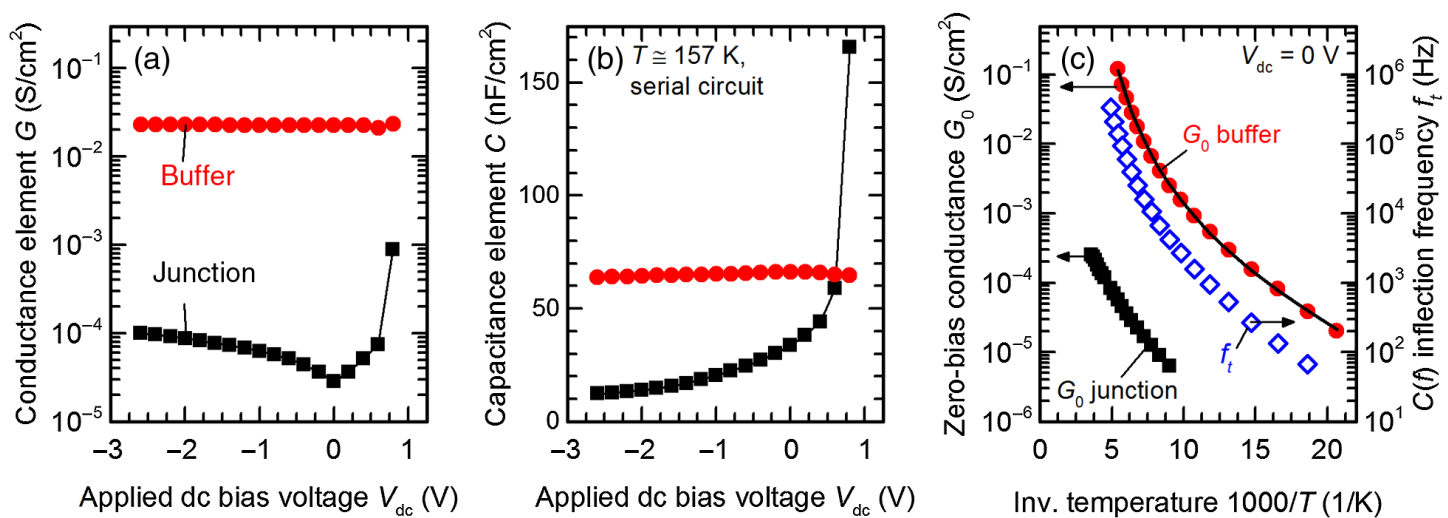

FIG. 6. (a) Conductance $G$ and (b) capacitance $C$ of two different $G$ - $C$ elements (black squares and red circles, respectively) derived from the impedance spectra in Fig. 5 as a function of applied dc bias voltage. The sample temperature is $T \approx 157 \mathrm{~K}$. Lines are guides to the eye. (c) Temperature dependence of the zero-bias conductance $G_{0}$ (left axis) of the buffer (red circles) and junction (black squares) circuit elements compared to the temperature-dependent inflection frequency $f_{t}$ of the main capacitance step (right axis). The solid black line is a fit to the quantum-tunneling model of Ref. [33].

feature is located around a characteristic frequency of $70 \mathrm{kHz}$ and is virtually unaffected by the applied bias voltage. We obtain similar trends for many individual devices from different processing runs (not shown here), including some containing a $\mathrm{Zn}(\mathrm{O}, \mathrm{S})$ buffer layer instead of the typical CdS buffer layer. We, thus, expect this set of data to be representative of typical $\mathrm{Cu}(\mathrm{In}, \mathrm{Ga}) \mathrm{Se}_{2}$ thin-film solar cells. A priori we do not know the origin of these two features, and we discuss both the serial electrical equivalent circuit and the classical defect model.

\section{Serial equivalent circuit: Junction and buffer layer}

For a buffer layer on top of the absorber, we use the serial equivalent circuit in Fig. 2(b). Employing Eqs. (1) and (12) for each of the two features in the admittance spectra, as we discuss in Sec. III A, allows us to estimate the individual conductance and capacitance values of both $G$ - $C$ elements, which are plotted in Figs. 6(a) and 6(b) as a function of applied bias voltage. The black squares in Fig. 6 correspond to the low-frequency feature identified in Fig. 5, while the red circles represent the constant feature at $70 \mathrm{kHz}$. We attribute the low-frequency feature (dotted line in Fig. 5 and black squares in Fig. 6) to the $p$ - $n$ junction of the device based on a number of considerations:

(i) The capacitance increases toward positive-bias voltage, as is expected for the capacitance of the space-charge region. The absolute capacitance values and their bias dependence are consistent with an absorber dopant concentration of the order of $10^{16} \mathrm{~cm}^{-3}$, typical for $\mathrm{Cu}(\mathrm{In}, \mathrm{Ga}) \mathrm{Se}_{2}$ solar cells. Note that according to the predictions in Sec. II B, the junction capacitance is close to the low-frequency limit of the measured effective capacitance and differs substantially from the highfrequency limit one usually chooses to calculate the dopant concentration from a Mott-Schottky plot. (ii) The zero-bias conductance is 3 orders of magnitude lower compared to the high-frequency feature, and the drastic increase in capacitance and conductance at the highest bias voltage of $+0.8 \mathrm{~V}$ reflects carrier injection. The low-frequency feature must accordingly be responsible for the diode character of the solar cell.

(iii) The increase of conductance in reverse bias agrees well with the slightly non-Ohmic nature of shunt currents typical for thin-film solar cells [29,30]. Our data suggest a voltage-dependent shunt current of approximately $I_{\mathrm{sh}} \propto|V|^{1.5}$, in a similar range as reported earlier [31,32].

The high-frequency feature (arrow in Fig. 5 and red circles in Fig. 6), on the other hand, is completely unaffected by variations of the bias voltage. For a buffer layer, we indeed do not expect to observe any significant voltage dependence in this experiment: since the dc conductance of the buffer layer greatly exceeds the conductance across the junction, the potential drop across the buffer layer will amount only to a very small fraction of the externally applied bias voltage. Any actual voltage dependence of the buffer conductance or capacitance, thus, becomes apparent only for much larger applied bias voltages.

Figure 6(c) shows the conductance $G_{0}$ of the buffer and junction elements at zero-bias voltage (left axis) and the inflection frequency $f_{t}$ of the main capacitance step (right axis) as a function of the inverse temperature $1000 / T$. The solid black line is a fit to the temperature-dependent buffer conductance based on Ref. [33]; see Appendix B. Both y axes of Fig. 6(c) are scaled similarly, and the buffer conductance and inflection frequency accordingly indeed exhibit a very similar temperature dependence as expected from Eq. (8). Note that the inflection frequencies shown in Fig. 6(c) differ slightly from earlier measurements shown in Fig. 4, presumably due to diffusion processes changing the 
TABLE I. Capacitance-thickness relation of the buffer-layer $G-C$ element.

\begin{tabular}{lccc}
\hline \hline Buffer layer & $\begin{array}{c}\text { Nominal } \\
\text { thickness }(\mathrm{nm})\end{array}$ & $\begin{array}{c}\text { Capacitance } \\
\left(\mathrm{nF} / \mathrm{cm}^{2}\right)\end{array}$ & $\begin{array}{c}\text { Max relative } \\
\text { permittivity } \varepsilon_{r}\end{array}$ \\
\hline $\mathrm{CdS}$ & 50 & $50-90$ & $3-5$ \\
$\mathrm{CdS}$ (double layer) & 100 & 90 & 10 \\
$\mathrm{Zn}(\mathrm{O}, \mathrm{S})$ & 35 & $130-230$ & $5-9$ \\
\hline \hline
\end{tabular}

transport properties of the buffer layer over time [34,35]. This change over time further supports a relation between the inflection frequency of the capacitance step and the transport properties of the buffer layer.

Our assumption that the high-frequency feature is related to the $\mathrm{CdS}$ buffer layer raises one issue regarding the extracted capacitance values $C_{b}$ assigned to the buffer layer: if the buffer layer is fully depleted, this capacitance is simply given by its geometrical capacitance $C_{b}=\varepsilon_{0} \varepsilon_{r} / d$, where $\varepsilon_{0}$ and $\varepsilon_{r}$ are the vacuum and relative dielectric permittivity, respectively, and $d$ is the buffer-layer thickness. If the buffer layer contains free charges, its capacitance increases. Table I summarizes capacitance values of the high-frequency feature together with the approximate nominal thickness of the corresponding buffer layer. The last column shows the estimated maximum relative permittivity $\varepsilon_{r}$ so that the geometrical capacitance calculated from the nominal layer thickness always remains smaller than the measured buffer-layer capacitance.

The relative dielectric permittivity is typically around $\varepsilon_{r} \approx 10$ for most bulk semiconductors and, thus, not fully compatible with the maximum values given in Table I, in particular for thin CdS films. Several authors, however, indeed report a decrease of $\varepsilon_{r}$ for thin layers of $\mathrm{CdS}$ [36-38]. In addition, elemental intermixing [39-43] and changes in dopant concentration [34,35] at the absorberbuffer interface might create an effective barrier layer that is, in fact, wider than the nominal thickness of the asdeposited CdS layer alone. In that case, measured bufferlayer capacitances will also be consistent with higher values of $\varepsilon_{r}$. Further note that we treat the $\mathrm{CdS}$ buffer separate from the $\mathrm{ZnO}$ window layer, and the interactions between those two layers might also complicate the actual composition at the various interfaces in the device.

\section{Parallel equivalent circuit: Junction and defect}

The main capacitance step can also result from a deep defect level rather than the buffer layer, and we explore which properties such a potential defect must have to be consistent with the experimental impedance spectra. One major distinction exists between defects and interlayers concerning the impedance spectroscopy: although both models result in an identical frequency dependence of the capacitance, their impact on the device impedance is notably different. A buffer layer is connected in series with

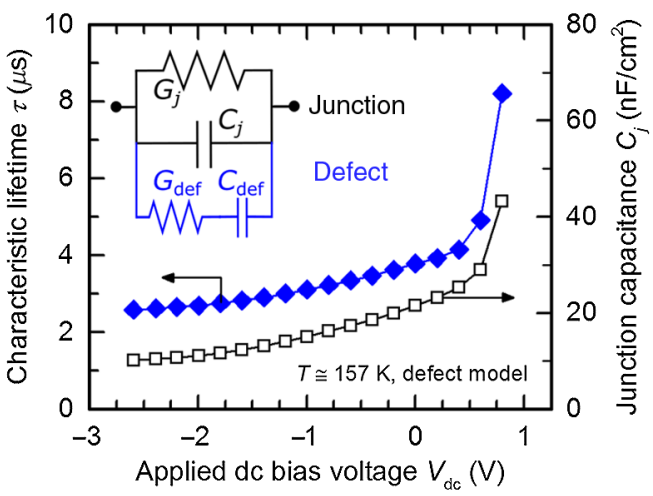

FIG. 7. Characteristic lifetime $\tau=C_{\text {def }} / G_{\text {def }}=\left(2 \pi f_{t}\right)^{-1}$ of a potential defect level (blue diamonds, left axis) and junction capacitance $C_{j}$ (open black squares, right axis) derived from the impedance spectra in Fig. 4. The sample temperature is $T \approx 157 \mathrm{~K}$. The inset shows the corresponding parallel equivalent circuit.

the junction [Fig. 2(b)] and, thus, adds its individual characteristic peak in the impedance spectrum. Changing the $G-C$ parameters of the junction by applying a bias voltage, thus, affects only one peak of the impedance spectrum, and the characteristic peak of the buffer layer remains unchanged. The capacitance and recombination resistance of a defect, however, are connected in parallel to the junction (see inset of Fig. 7), and the two resulting impedance peaks are, thus, no longer independent. For an arbitrary defect level, the high-frequency feature in Fig. 5 will, thus, also be expected to shift with applied bias voltage. It is certainly possible to envision a defect where the highfrequency feature does not shift with applied bias voltage: if the frequency response of this potential defect level changes in precise correlation with the junction parameters, the differences in the recombination dynamics of the defect at different bias voltages might just exactly cancel the bias dependence of the junction. Such a perfect balance of the bias dependences of junction and defect parameters, however, requires a very specific combination of defect concentration and location within the absorber, energetic depth of the defect level, and its capture cross sections.

For a discreet defect level within the bulk of the CIGS absorber, we indeed expect a certain correlation between the frequency response of the junction and defects: defects change their charge state only if the majority-carrier Fermi level crosses the defect level, and this condition is fulfilled only at a certain depth directly linked to the space-charge region width $[1,28]$. To test whether such defects can realistically explain our measurements, we fit the experimental impedance spectra shown in Fig. 5 with the electrical equivalent circuit shown in the inset of Fig. 7, which includes a defect level with capacitance $C_{\text {def }}$ and characteristic lifetime $\tau=C_{\mathrm{def}} / G_{\mathrm{def}}$ in parallel to the junction. Comparing the apparent depth calculated from the junction capacitance $C_{j}$ and the total capacitance 
$C_{j}+C_{\mathrm{def}}$ (junction plus defect), we obtain a constant difference of 140-150 $\mathrm{nm}$ independent of bias voltage. Note that this is consistent with a discrete bulk defect but excludes interface defects where the bias dependence of the defect capacitance instead depends on the energetic defect distribution within the absorber band gap.

The recombination dynamics of a discreet defect level are described by the characteristic lifetime $\tau=C_{\mathrm{def}} / G_{\mathrm{def}}$, which is identical to the characteristic time constant of the corresponding capacitance step $\tau=\left(2 \pi f_{t}\right)^{-1}$. Note that $f_{t}$ is the inflection frequency of the capacitance step and is generally different from both of the characteristic frequencies of the impedance spectrum. The blue diamonds in Fig. 7 show the bias dependence of the characteristic lifetime $\tau$ required to reproduce the bias-independent high-frequency feature in the impedance spectra of Fig. 5. This behavior is highly peculiar for a defect: Eq. (11) predicts a constant value of $\tau$ unless either capture cross section or the energetic depth of the defect level change with applied bias voltage or vary with depth into the absorber. Furthermore, the characteristic lifetime follows a similar bias dependence as the junction capacitance (open black squares in Fig. 7), and, in fact, both parameters are strongly correlated: the characteristic lifetime is empirically identical to $\tau=1.5 \mu \mathrm{s}+$ $0.1\left(\mu \mathrm{sm}^{2} / \mathrm{nF}\right) C_{j}$ for voltages up to $+0.4 \mathrm{~V}$. Although a defect level with such peculiar properties can, in principle, exist, it appears unlikely that defects are responsible for the capacitance step observed here.

\section{Capacitance spectra under illumination}

Based on our model presented in Sec. II, in particular Eq. (8), we expect the inflection frequencies of the main capacitance step to depend critically on the conductivity in parts of the solar cell. We test this hypothesis by illuminating a solar cell through an optical port in the cryostat fixed with a neutral density filter wheel assembly to adjust the illumination intensity. We use two LEDs (see Appendix C) with different spectra to compare dominant absorption in the buffer layer and absorber:

(i) A UV LED with peak wavelength of $365 \mathrm{~nm}$ (FWHM of $8 \mathrm{~nm}$ ) corresponding to a photon energy of $3.4 \mathrm{eV}$ and, thus, predominantly absorbed in the CdS buffer layer.

(ii) An IR LED with peak wavelength of $940 \mathrm{~nm}$ (FWHM of $65 \mathrm{~nm}$ ) corresponding to a photon energy of $1.3 \mathrm{eV}$ well below the expected band gap of $E_{g} \approx 2.4 \mathrm{eV}$ of the CdS buffer layer.

Note that the device used for this experiment contains a double layer of $\mathrm{CdS}$ in order to increase the nominal thickness of the CdS and, thus, enhance absorption of the UV illumination within the buffer layer. We do not expect any fundamental differences due to the thicker CdS layer, and we verify results similar to those presented below using white-light illumination of standard devices with a single CdS layer. All illuminated admittance spectroscopy

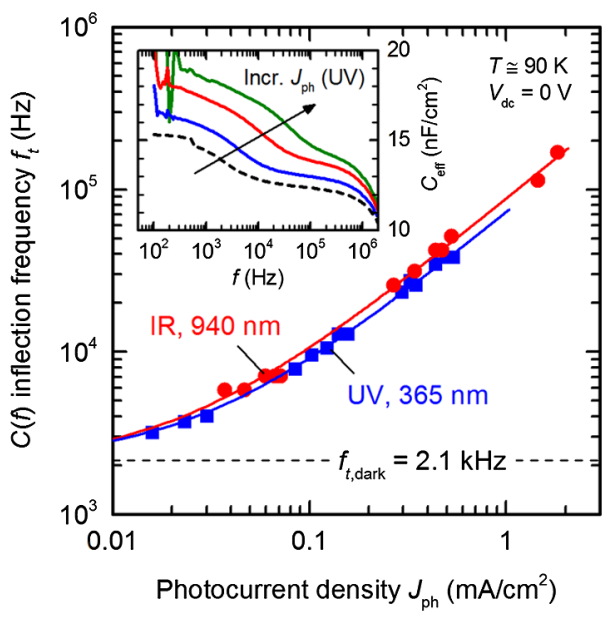

FIG. 8. Inflection frequency $f_{t}$ of the main capacitance step as a function of photocurrent density $J_{\mathrm{ph}}$ at a sample temperature of $T \approx 90 \mathrm{~K}$ for illumination with 365-nm (UV, blue squares) and 940-nm (IR, red circles) light. Lines are linear fits to the data. Inset: Exemplary capacitance spectra $C(f)$ in the dark (black dashed line) and for different UV illumination intensities (solid lines, $J_{\mathrm{ph}} \approx 30,150$, and $500 \mu \mathrm{A} / \mathrm{cm}^{2}$ ).

measurements are nominally performed at zero-bias voltage. The actual dc voltage across the device deviates from ideal short-circuit conditions due to the finite input resistance of the impedance meter, but the deviations are negligible (below $40 \mathrm{mV}$ ) for all measurements presented here. We, thus, assume that the generated photocurrent is equal to the measured dc current through the device. It is worth pointing out that we do not observe any distortion of the current-voltage curves [44] at any temperature upon IR illumination. We also do not observe any metastable effects and always obtain the same capacitance spectrum at a given illumination condition, independent of the previous illumination history of the sample.

Independent of the wavelength of illumination, we find that the main capacitance step shifts to higher frequencies upon illumination at all temperatures. This behavior is illustrated in Fig. 8, which shows the inflection frequency of the main capacitance step as a function of the photocurrent density $J_{\mathrm{ph}}$ for both UV and IR LEDs for a chosen sample temperature of $T \approx 90 \mathrm{~K}$. Exemplary capacitance spectra for different UV illumination intensities are shown in the inset. In the dark, we obtain an inflection frequency of the main capacitance step of $f_{t, \text { dark }}=2.1 \mathrm{kHz}$, which shifts over 2 orders of magnitude upon illumination. Inflection frequencies in admittance spectroscopy can typically be resolved only over roughly 3 orders of magnitude, taking into account low-frequency noise (e.g., low phase angle of the complex impedance) and high-frequency features (e.g., series resistance). The illumination-induced shift of the inflection frequency reported here, thus, represents a significant portion of the frequency range accessible in experiment. Indeed, when increasing 
the illumination intensity further, we find that the main capacitance step merges with a high-frequency capacitance step caused by series resistance and completely disappears at all temperatures.

Despite small differences between UV and IR illumination, the two sets of data shown in Fig. 8 behave fairly similarly. Note that most of the UV light is absorbed in the $\mathrm{CdS}$ buffer layer or $\mathrm{ZnO}$ window layer and does not contribute to the photocurrent. Accordingly, a significantly higher illumination intensity - approximately by a factor of 90-is required for the UV LED to yield the same photocurrent as the IR LED and, thus, obtain a similar shift in inflection frequency. We conclude that the inflection frequency of the main capacitance step is sensitive to the number of extracted photogenerated carriers (photocurrent) not to the number of absorbed photons (illumination intensity). Note that the photocurrent densities here are up to 3 orders of magnitude lower compared to standard 1-sun illumination, and we can, thus, neglect any highinjection effects.

The shift toward higher inflection frequencies with increasing illumination intensity appears to follow a linear trend, as suggested by the linear fits represented by solid lines in Fig. 8. Within the framework of the standard defect model expressed by Eq. (11), such a behavior is peculiar and either requires a (linearly) higher capture cross section or (logarithmically) more shallow activation energy of the defect with increasing photocarrier density. Similar changes of the capacitance spectrum under white-light illumination have been reported previously [45], and the authors argued that trapping effects in exponential band tails might result in the required defect properties. Our biasdependent measurements in Sec. III B 2 demonstrate, however, that the main charge response of a potential defect is located 140-150 $\mathrm{nm}$ within the space-charge region, and a significant number of defects, thus, need to be energetically deeper than the bulk Fermi level. For an exponential band tail, we rather expect the main charge response to occur near the space-charge edge, where the number of defects crossing the Fermi level is largest.

For a transport barrier due to a depleted buffer layer, on the other hand, such a linear increase shown in Fig. 8 is readily understood by assuming a photoconductive contribution to the buffer-layer conductance $G_{b}$ in Eq. (8), i.e., $G_{b}=G_{b, \text { dark }}+\Delta G\left(J_{\mathrm{ph}}\right)$. The striking similarity between UV and IR illumination concerning the correlation with photocurrent density implies that the injection of photoexcited electrons from the absorber into the CdS buffer layer plays a crucial role, not just photoexcitation within the $\mathrm{CdS}$ buffer layer itself. This observation appears reasonable for typical thin-film solar cells: the presence of photoexcited holes in the $n$-type CdS buffer layer might increase recombination, particularly in conjunction with potentially high defect concentrations within the CdS buffer layer or sputter damage at the $\mathrm{CdS} / \mathrm{ZnO}$ interface. In contrast, if
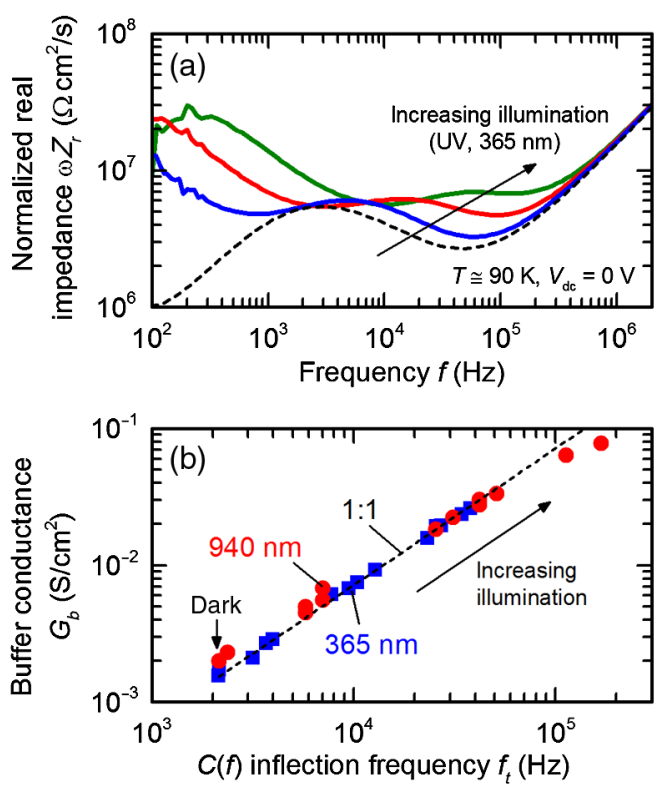

FIG. 9. (a) Exemplary normalized real impedance spectra $\omega Z_{r}(f)$ in the dark (black dashed line) and for different UV illumination intensities (solid lines, $J_{\mathrm{ph}} \approx 30,150$, and $500 \mu \mathrm{A} / \mathrm{cm}^{2}$ ) at $T \approx 90 \mathrm{~K}$. (b) Conductance $G_{b}$ of the bufferlayer $G-C$ element extracted from the impedance spectra plotted as a function of inflection frequency $f_{t}$ of the main capacitance step for 365-nm (UV, blue squares) and 940-nm (IR, red circles) illumination. The dashed line represents a 1:1 correlation; dark measurements are indicated by a black arrow.

photoexcitation dominantly occurs in the absorber layer, holes will be kept away from the CdS buffer layer due to the large valence-band offset at the CIGS/CdS interface. For our interpretation of the capacitance step, however, the exact mechanism of photoconductivity in the $\mathrm{CdS}$ buffer layer is not relevant.

Any change in the buffer conductance due to photoexcited or injected carriers should be visible in the corresponding impedance spectra. Figure 9(a) shows four exemplary spectra of the normalized real impedance $\omega Z_{r}(f)$ in the dark (black dashed line) and for 365-nm (UV) illumination intensities corresponding to photocurrent densities of $J_{\mathrm{ph}} \approx 30 \mu \mathrm{A} / \mathrm{cm}^{2}$ (blue), $150 \mu \mathrm{A} / \mathrm{cm}^{2}$ (red), and $500 \mu \mathrm{A} / \mathrm{cm}^{2}$ (green). These spectra correspond to the capacitance spectra shown in the inset of Fig. 8. At the chosen sample temperature of $T \approx 90 \mathrm{~K}$ and in the dark, the characteristic frequency of the junction $G-C$ element is well below our measurement range, and we resolve only the buffer $G-C$ element with characteristic frequency of $3 \mathrm{kHz}$ (the increase in $\omega Z_{r}$ at high frequencies is caused by series resistance). Upon illumination, we observe significant changes in the impedance spectra:

(i) The impedance peak of the junction shifts into the measurement range at low frequencies, which indicates that the junction parameters, as expected, change drastically in the presence of photoexcited 
carriers. In most cases, we still cannot quantitatively and reliably resolve this feature, however, due to low-frequency noise in the measurements.

(ii) The impedance peak associated with the buffer layer shifts to higher frequencies upon illumination, in agreement with our model presented in the previous paragraphs.

A similar peak height for the buffer-layer $G-C$ element for all illumination intensities implies that the buffer capacitance remains approximately constant; compare Eq. (12). Thus, the corresponding buffer conductance must increase with illumination to explain the shift of the characteristic peak in the impedance spectra, since the characteristic frequency $f_{\text {char }}$ of any $G-C$ element is given by $2 \pi f_{\text {char }}=1 / \tau=G / C$. The conductance $G_{b}$ of the buffer-layer $G-C$ element obtained in this way for all illumination intensities is plotted in Fig. 9(b) as a function of the inflection frequency $f_{t}$ of the corresponding main capacitance step shown in Fig. 8. The buffer conductance starts at $G_{b}=(1.5 \pm 0.5) \mathrm{mS} / \mathrm{cm}^{2}$ in the dark [black arrow in Fig. 9(b)] and clearly shifts over almost 2 orders of magnitude upon illumination, similar to the inflection frequency of the main capacitance step. Agreement with a perfect $1: 1$ correlation indicated by the black dashed line in Fig. 9(b) is excellent, particularly at low and intermediate illumination intensities. Note that we neglect any illumination-induced changes in the junction parameters or buffer-layer capacitance, effects which might further contribute to the shift of the capacitance step especially at high illumination intensities and explain small deviations from a perfect 1:1 correlation in Fig. 9(b).

We conclude that the illumination-induced shift of the main capacitance step in admittance spectroscopy for this device is almost exclusively attributed to an increased conductivity in the buffer layer, presumably due to the presence of injected photogenerated electrons. Consequently, the occurrence of the main capacitance step in the dark must also be attributed to the presence of the buffer layer, and at least in our case, is not related to any defect.

\section{INTERPRETATION OF ADMITTANCE MEASUREMENTS}

The origin of the $N 1$ signature commonly observed in temperature-dependent capacitance spectra of $\mathrm{Cu}(\mathrm{In}, \mathrm{Ga}) \mathrm{Se}_{2}$ thin-film solar cells has been discussed already for decades. Bulk defects, interface defects, or transport barriers have been suggested previously as the most likely candidates for the $N 1$ signature. In Sec. II, we quantify the effect of a buffer layer on the device admittance and impedance spectra by modeling the buffer layer as a conductance-capacitance $(G-C)$ element in series with the $p-n$ junction of the solar cell. We demonstrate that each serial $G$ - $C$ element in the electrical equivalent circuit of the device contributes a characteristic step and peak, respectively, to the real and imaginary parts of the frequency-dependent complex device impedance. As a consequence, the presence of a buffer layer also results in a characteristic step in the capacitance spectrum of devices containing such a buffer layer, even if the individual conductance and capacitance values of each circuit element are independent of frequency. In fact, the dominant dependence of inflection frequency on buffer-layer properties can naturally explain the universality of the $N 1$ level in CIGS solar cells.

For a series connection of two $G-C$ elements (here, the buffer layer and junction), the inflection frequency of the resulting capacitance step, in general, depends on all circuit parameters, while the individual characteristic frequencies of the impedance spectrum are indeed characteristic of the respective circuit element. Then, the position of a particular feature in the impedance spectrum does not depend on the properties of any other layers also present in the device, and we can study the behavior of individual layers independently. Since the buffer layer is connected in series with the junction, the characteristic peak of the buffer layer is unaffected by changes to the $p-n$ junction, e.g., by applying a bias voltage, and vice versa.

The main capacitance step can also result from a deep defect level rather than the buffer layer since both models result in an identical frequency dependence of the capacitance. Contrary to the buffer layer, however, the circuit elements (capacitance and recombination resistance) describing a recombination-active deep defect level are connected in parallel to the junction, and the two resulting impedance peaks are no longer independent. We can, thus, distinguish between the capacitance steps caused by a buffer layer or by deep defects by studying the evolution of the individual characteristic peaks in the impedance spectrum in response to external stimuli, e.g., bias voltage or illumination. In particular, if only one of the two characteristic impedance peaks shifts with excitation, we can derive strict constraints for the recombination parameters of a potential deep defect level or possibly rule out defects entirely.

In order to clarify the origin of the main capacitance step in $\mathrm{Cu}(\mathrm{In}, \mathrm{Ga}) \mathrm{Se}_{2}$ thin-film solar cells, its inflection frequency is investigated as a function of applied bias voltage and illumination intensity. Two distinct features are clearly resolved in the bias-dependent impedance spectra (Fig. 5). One of these features agrees well with current-voltage and capacitance-voltage characteristics expected for the $p$ - $n$ junction of a thin-film solar cell, while the second feature in the impedance spectra is independent of the applied bias voltage (Fig. 6). Upon illumination, the inflection frequency of the main capacitance step shifts to higher frequencies, proportional to the measured photocurrent density across the device (Fig. 8). The shift in inflection frequency is shown to be mainly driven by an increased conductance of the bias-independent $G-C$ element (Fig. 9). We find that deep defects within the absorber need to feature some peculiar properties to explain a bias-independent inflection frequency proportional to the photocurrent: their capture 
cross section and energetic separation from the valence band need to change with varying space-charge region capacitance and illumination in a precisely determined way. In contrast, all experimental observations follow naturally for a transport barrier caused by a buffer layer in the device, since the inflection frequency of the corresponding capacitance step is then directly related to the buffer conductance. Specifically, the voltage across the buffer layer is typically small due to its high conductance compared to the junction, and photoexcited carriers directly increase the buffer conductance.

\section{CONCLUSIONS}

In conclusion, we show that impedance spectroscopy is a powerful tool to study specific layers within the complex device structure of typical thin-film solar cells, in particular, if bias voltage and illumination are exploited as additional experimental variables. Employing this technique, we find that the main capacitance step observed in admittance spectroscopy of $\mathrm{Cu}(\mathrm{In}, \mathrm{Ga}) \mathrm{Se}_{2}$ thin-film solar cells, commonly referred to as the $N 1$ signature, is most plausibly explained by the presence of a buffer layer connected in series to the $p-n$ junction of the device. Because of the similarity in device concept, we expect that this observation equally holds true for other thin-film technologies incorporating a buffer layer in the device stack.

\section{ACKNOWLEDGMENTS}

The authors like to thank A. Torello for scrutinizing the procedures used to deconvolute the impedance spectra and M. Melchiorre and T. Bertram for assistance in sample preparation. This study is funded by the Fonds National de la Recherche Luxembourg for the project "Surface passivation for thin film photovoltaics (SURPASS)."

\section{APPENDIX A: DECONVOLUTION OF IMPEDANCE SPECTRA}

As we discuss in Sec. III A, we determine the parameters of the equivalent circuit by peak analysis and numerical least-squares regression. Figure 10 shows a comparison of the measured capacitance (solid symbols, same data as Fig. 5) to fitting results (solid black lines) for an equivalent circuit consisting of two $G-C$ elements (buffer layer and junction) and a lumped series resistance $\left(0.6-0.8 \Omega \mathrm{cm}^{2}\right.$ at this temperature). The modeled capacitance usually does not perfectly reproduce the slope of the experimental capacitance step or a slight capacitance dispersion at low frequencies. This deviations can be reduced by replacing the buffer-layer $G$ - $C$ element with a constant phase element (CPE) [admittance $Y=(i \omega)^{\alpha} Q$; fit results shown by pink dashed lines in Fig. 10], which might be an indication of inhomogeneities within the buffer layer [46].

The conductance and capacitance parameters of the circuit elements obtained by both fitting procedures are

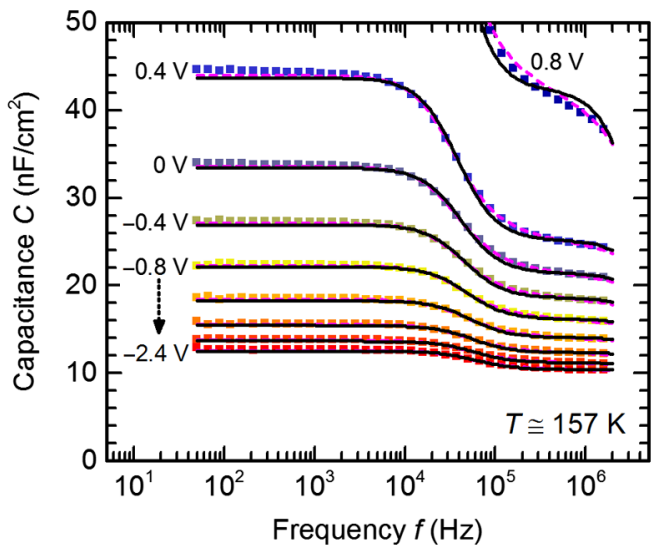

FIG. 10. Experimental capacitance (solid symbols) and effective capacitance resulting from a least-squares fit treating the buffer layer as a $G-C$ element (solid black lines) or CPE element (pink dashed lines).

compared to the peak analysis in Fig. 11 (solid symbols, peak analysis; crosses, fitting results). We use the same formalism as Sugiyama et al.-Eq. (2) in Ref. [24]—-to convert the CPE parameters to an equivalent capacitance $(\alpha=0.95 \pm 0.01$ for this set of data). As shown in Fig. 11, the junction parameters are virtually unaffected by the choice of analysis. The buffer-layer conductance differs by less than $20 \%$ and the capacitance by less than $15 \%(<10 \%$ for $V_{\mathrm{dc}}>-1.5 \mathrm{~V}$, where the peak overlap is less severe; see Fig. 5).

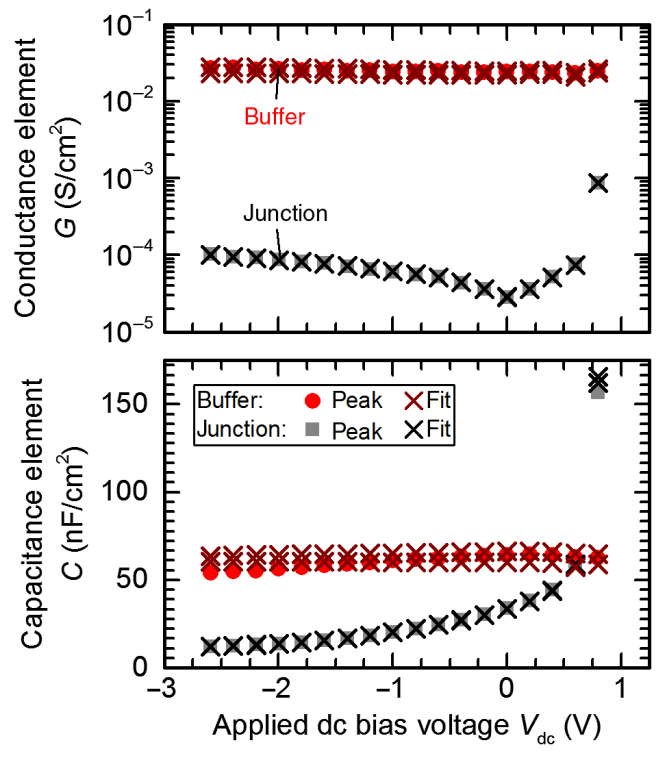

FIG. 11. Conductance (top) and capacitance (bottom) of the buffer layer (dark red and light red) and junction (black and gray) circuit elements as a function of applied voltage at $T \approx 157 \mathrm{~K}$. Values determined by peak analysis (solid symbols) or leastsquares regression (crosses). Two different fits are shown, where the buffer element is represented either by a $G-C$ or CPE element (lower conductance and higher capacitance values correspond to the CPE fit). 


\section{APPENDIX B: TEMPERATURE-DEPENDENT BUFFER CONDUCTANCE}

The temperature-dependent conductance of the buffer layer often does not follow an ideal thermally activated behavior, as, for example, shown in Fig. 6(c). Instead, we find an excellent agreement with the quantum-tunneling model across a vibrating barrier proposed by Hurd [33], where the temperature-dependent conductance is of the form

$$
G(T) \propto \frac{1}{T} \exp \left(-\frac{U}{k T}\right) \exp \left(\frac{k T}{A}\right)
$$

with a "coincidence" term determined by the parameter $U$ and a "tunneling" term defined by $A$. The fit (solid black line) in Fig. 6(c) yields $U=20.1 \mathrm{meV}$ and $A=1.85 \mathrm{meV}$ for this particular $\mathrm{CdS}$ buffer layer. We find similar values for most of our measurements, with $U \approx 18-35 \mathrm{meV}$ and $A \approx 1.5-2.3 \mathrm{meV}$ for $\mathrm{CdS}$ and $1.1-1.9 \mathrm{meV}$ for $\mathrm{Zn}(\mathrm{O}, \mathrm{S})$ buffer layers. Despite the similar temperature dependence, the proportionality constant in Eq. (B1) and, thus, the absolute conductance, might differ by up to 2 orders of magnitude between different devices. Furthermore, these values are likely specific to the buffer layers deposited in our lab, since, for example, in a recent study we reported a thermally activated behavior for devices fabricated at different institutes [20]. For the temperature-dependent conductance of the $p-n$ junction also shown in Fig. 6(c), we find an activation energy of $E_{a}=50-80 \mathrm{meV}$ (or, alternatively, $U=51.6 \mathrm{meV}$ and $A=10.2 \mathrm{meV}$ ).

\section{APPENDIX C: LIGHT SOURCES FOR ILLUMINATION}

For the illuminated measurements in Sec. III C, collimated high-power LEDs with approximate total beam power and peak wavelength of $60 \mathrm{~mW}, 365 \mathrm{~nm}$ (UV)

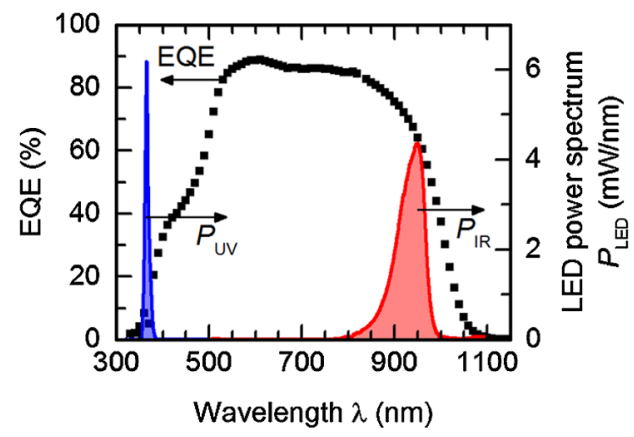

FIG. 12. Left axis: Wavelength dependence of the EQE (black squares) of the $\mathrm{Cu}(\mathrm{In}, \mathrm{Ga}) \mathrm{Se}_{2}$ solar cell with double $\mathrm{CdS}$ layer used for the illumination study in Sec. III C. Right axis: Nominal light power $P_{\text {LED }}$ according to the data sheets of the UV (blue line) and IR (red line) LED as a function of the wavelength. and $320 \mathrm{~mW}, 940 \mathrm{~nm}$ (IR) are used in conjunction with a set of neutral density filters. The power spectra of both LEDs are shown in Fig. 12 together with the external quantum efficiency of the solar cell used for this experiment. The band gap of the $\mathrm{Cu}(\mathrm{In}, \mathrm{Ga}) \mathrm{Se}_{2}$ absorber is $1.18 \mathrm{eV}$ as determined by linear extrapolation of the longwavelength external quantum efficiency (EQE).

[1] P. Blood and J. W. Orton, The Electrical Characterization of Semiconductors: Majority Carriers and Electron States (Academic Press, London, 1992).

[2] E. H. Nicollian and J. R. Brews, MOS (Metal Oxide Semiconductor) Physics and Technology (Wiley, New York, 1982).

[3] C.-T. Sah, Detection of recombination centers in solar cells from junction capacitance transients, IEEE Trans. Electron Devices 24, 410 (1977).

[4] T. Walter, R. Herberholz, C. Müller, and H. W. Schock, Determination of defect distributions from admittance measurements and application to $\mathrm{Cu}(\mathrm{In}, \mathrm{Ga}) \mathrm{Se}_{2}$ based heterojunctions, J. Appl. Phys. 80, 4411 (1996).

[5] T. P. Weiss, A. Redinger, D. Regesch, M. Mousel, and S. Siebentritt, Direct evaluation of defect distributions from admittance spectroscopy, IEEE J. Photovoltaics 4, 1665 (2014).

[6] D. A. R. Barkhouse, O. Gunawan, T. Gokmen, T. K. Todorov, and D. B. Mitzi, Device characteristics of a $10.1 \%$ hydrazine-processed $\mathrm{Cu}_{2} \mathrm{ZnSn}(\mathrm{Se}, \mathrm{S})_{4}$ solar cell, Prog. Photovoltaics 20, 6 (2012).

[7] M. Burgelman and P. Nollet, Admittance spectroscopy of thin film solar cells, Solid State Ionics 176, 2171 (2005).

[8] Y. Y. Proskuryakov, K. Durose, B. M. Taele, and S. Oelting, Impedance spectroscopy of unetched $\mathrm{CdTe} / \mathrm{CdS}$ solar cells-Equivalent circuit analysis, J. Appl. Phys. 102, 024504 (2007).

[9] R. Herberholz, M. Igalson, and H. W. Schock, Distinction between bulk and interface states in $\mathrm{CulnSe}_{2} / \mathrm{CdS} / \mathrm{ZnO}$ by space charge spectroscopy, J. Appl. Phys. 83, 318 (1998).

[10] A. Krysztopa, M. Igalson, L. Gütay, J. K. Larsen, and Y. Aida, Defect level signatures in $\mathrm{CuInSe}_{2}$ by photocurrent and capacitance spectroscopy, Thin Solid Films 535, 366 (2013).

[11] J. T. Heath, J. D. Cohen, and W. N. Shafarman, Bulk and metastable defects in $\mathrm{CuIn}_{(1-x)} \mathrm{Ga}_{(x)} \mathrm{Se}_{2}$ thin films using drive-level capacitance profiling, J. Appl. Phys. 95, 1000 (2004).

[12] U. Rau, D. Braunger, R. Herberholz, H. W. Schock, J.-F. Guillemoles, L. Kronik, and D. Cahen, Oxygenation and air-annealing effects on the electronic properties of $\mathrm{Cu}(\mathrm{In}, \mathrm{Ga}) \mathrm{Se}_{2}$ films and devices, J. Appl. Phys. 86, 497 (1999).

[13] U. Reisloehner, H. Metzner, and C. Ronning, Hopping Conduction Observed in Thermal Admittance Spectroscopy, Phys. Rev. Lett. 104, 226403 (2010).

[14] U. Reisloehner and C. Ronning, Maxwell-Wagner polarization in $\mathrm{Cu}(\mathrm{In}, \mathrm{Ga})(\mathrm{S}, \mathrm{Se})_{2}$, Appl. Phys. Lett. 100, 252111 (2012). 
[15] T. Eisenbarth, T. Unold, R. Caballero, C. A. Kaufmann, and H.-W. Schock, Interpretation of admittance, capacitancevoltage, and current-voltage signatures in $\mathrm{Cu}(\mathrm{In}, \mathrm{Ga}) \mathrm{Se}_{2}$ thin film solar cells, J. Appl. Phys. 107, 034509 (2010).

[16] M. Igalson, A. Urbaniak, and M. Edoff, Reinterpretation of defect levels derived from capacitance spectroscopy of CIGSe solar cells, Thin Solid Films 517, 2153 (2009).

[17] J. Lauwaert, L. Callens, S. Khelifi, K. Decock, M. Burgelman, A. Chirila, F. Pianezzi, S. Buecheler, A. N. Tiwari, and H. Vrielinck, About RC-like contacts in deep level transient spectroscopy and $\mathrm{Cu}(\mathrm{In}, \mathrm{Ga}) \mathrm{Se}_{2}$ solar cells, Prog. Photovoltaics 20, 588 (2012).

[18] J. Lauwaert, L. V. Puyvelde, J. Lauwaert, J. W. Thybaut, S. Khelifi, M. Burgelman, F. Pianezzi, A. N. Tiwari, and H. Vrielinck, Assignment of capacitance spectroscopy signals of CIGS solar cells to effects of non-Ohmic contacts, Sol. Energy Mater. Sol. Cells 112, 78 (2013).

[19] T. P. Weiss, S. Nishiwaki, B. Bissig, R. Carron, E. Avancini, J. Löckinger, S. Buecheler, and A. N. Tiwari, Injection current barrier formation for RbF postdeposition-treated $\mathrm{Cu}(\mathrm{In}, \mathrm{Ga}) \mathrm{Se}_{2}$-based solar cells, Adv. Mater. Interfaces 5, 1701007 (2018).

[20] F. Werner, M. H. Wolter, S. Siebentritt, G. Sozzi, S. D. Napoli, R. Menozzi, P. Jackson, W. Witte, R. Carron, E. Avancini, T. Weiss, and S. Buecheler, Alkali treatments of $\mathrm{Cu}(\mathrm{In}, \mathrm{Ga}) \mathrm{Se}_{2}$ thin-film absorbers and their impact on transport barriers, Prog. Photovolt. Res. Appl. (to be published).

[21] F. Werner, A. Zelenina, and S. Siebentritt, Experimental evidence for CdS-related transport barrier in thin film solar cells and its impact on admittance spectroscopy, in Proceedings of the 44th IEEE Photovoltaic Specialists Conference, Washington, D.C., 2017 (IEEE, New York, 2017), p. 709.

[22] S. S. Hegedus and W. N. Shafarman, Thin-film solar cells: Device measurements and analysis, Prog. Photovoltaics 12, 155 (2004).

[23] H. Bayhan and A.S. Kavasoglu, Study of CdS/ $\mathrm{Cu}(\mathrm{In}, \mathrm{Ga}) \mathrm{Se}_{2}$ heterojunction interface using admittance and impedance spectroscopy, Solar Energy 80, 1160 (2006).

[24] M. Sugiyama, M. Hayashi, C. Yamazaki, N. B. Hamidon, Y. Hirose, and M. Itagaki, Application of impedance spectroscopy to investigate the electrical properties around the $p n$ interface of $\mathrm{Cu}(\mathrm{In}, \mathrm{Ga}) \mathrm{Se}_{2}$ solar cells, Thin Solid Films 535, 287 (2013).

[25] M. Sugiyama, H. Sakakura, S.-W. Chang, and M. Itagaki, Investigation of sputtering damage around $p n$ interfaces of $\mathrm{Cu}(\mathrm{In}, \mathrm{Ga}) \mathrm{Se}_{2}$ solar cells by impedance spectroscopy, Electrochim. Acta 131, 236 (2014).

[26] G. Friesen, M. Özsar, and E. Dunlop, Impedance model for CdTe solar cells exhibiting constant phase element behaviour, Thin Solid Films 361-362, 303 (2000).

[27] Y. Y. Proskuryakov, K. Durose, M. K. Al Turkestani, I. Mora-Sero, G. Garcia-Belmonte, F. Fabregat-Santiago, J. Bisquert, V. Barrioz, D. Lamb, S. J. C. Irvine, and E. W. Jones, Impedance spectroscopy of thin-film CdTe/CdS solar cells under varied illumination, J. Appl. Phys. 106, 044507 (2009).

[28] J. Heath and P. Zabierowski, Capacitance spectroscopy of thin-film solar cells, in Advanced Characterization
Techniques for Thin Film Solar Cells, edited by D. Abou-Ras, T. Kirchartz, and U. Rau (Wiley-VCH, Weinheim, 2011).

[29] S. Dongaonkar, J. D. Servaites, G. M. Ford, S. Loser, J. Moore, R. M. Gelfand, H. Mohseni, H. W. Hillhouse, R. Agrawal, M. A. Ratner, T. J. Marks, M. S. Lundstrom, and M. A. Alam, Universality of non-Ohmic shunt leakage in thin-film solar cells, J. Appl. Phys. 108, 124509 (2010).

[30] A. Zelenina, F. Werner, H. Elanzeery, M. Melchiorre, and S. Siebentritt, Space-charge-limited currents in CIS-based solar cells, Appl. Phys. Lett. 111, 213903 (2017).

[31] J.-H. Tan and W. A. Anderson, Current transport in copper indium gallium diselenide solar cells comparing mesa diodes to the full cell, Sol. Energy Mater. Sol. Cells 77, 283 (2003).

[32] B. L. Williams, S. Smit, B. J. Kniknie, K. J. Bakker, W. Keuning, W. M. M. Kessels, R. E. I. Schropp, and M. Creatore, Identifying parasitic current pathways in CIGS solar cells by modelling dark $J-V$ response, Prog. Photovoltaics 23, 1516 (2015).

[33] C. M. Hurd, Quantum tunnelling and the temperature dependent DC conduction in low-conductivity semiconductors, J. Phys. C 18, 6487 (1985).

[34] F. Werner, F. Babbe, J. Burkhart, C. Spindler, H. Elanzeery, and S. Siebentritt, Interdiffusion and doping gradients at the buffer/window interface in thin-film solar cells (to be published).

[35] F. Werner, T. Bertram, J. Mengozzi, and S. Siebentritt, What is the dopant concentration in polycrystalline thin-film $\mathrm{Cu}(\mathrm{In}, \mathrm{Ga}) \mathrm{Se}_{2}$ ?, Thin Solid Films 633, 222 (2017).

[36] L. Lu, X.-L. Xu, W.-T. Liang, and H.-F. Lu, Raman analysis of $\mathrm{CdSe} / \mathrm{CdS}$ core-shell quantum dots with different $\mathrm{CdS}$ shell thickness, J. Phys. Condens. Matter 19, 406221 (2007).

[37] P. P. Sahay, R. K. Nath, and S. Tewari, Optical properties of thermally evaporated CdS thin films, Cryst. Res. Technol. 42, 275 (2007).

[38] F. Yakuphanoglu, C. Viswanathan, P. Peranantham, and D. Soundarrajan, Effects of the film thickness on optical constants of transparent $\mathrm{CdS}$ thin films deposited by chemical bath deposition, J. Optoelectron. Adv. Mater. 11, 945 (2009).

[39] D. Abou-Ras, G. Kostorz, A. Romeo, D. Rudmann, and A. N. Tiwari, Structural and chemical investigations of CBD- and PVD-CdS buffer layers and interfaces in $\mathrm{Cu}$ (In, Ga) $\mathrm{Se}_{2}$-based thin film solar cells, Thin Solid Films 480-481, 118 (2005).

[40] D. Liao and A. Rockett, Cd doping at the $\mathrm{CuInSe}_{2} / \mathrm{CdS}$ heterojunction, J. Appl. Phys. 93, 9380 (2003).

[41] T. Nakada and A. Kunioka, Direct evidence of Cd diffusion into $\mathrm{Cu}(\mathrm{In}, \mathrm{Ga}) \mathrm{Se}_{2}$ thin films during chemical-bath deposition process of CdS films, Appl. Phys. Lett. 74, 2444 (1999).

[42] K. Ramanathan, R. Noufi, J. Granata, J. Webb, and J. Keane, Prospects for in situ junction formation in $\mathrm{CuInSe}_{2}$ based solar cells, Sol. Energy Mater. Sol. Cells 55, 15 (1998).

[43] B. Ümsür, W. Calvet, B. Höpfner, A. Steigert, I. Lauermann, M. Gorgoi, K. Prietzel, H. A. Navirian, C. A. Kaufmann, T. Unold, and M. C. Lux-Steiner, Investigation of Cu-poor and $\mathrm{Cu}$-rich $\mathrm{Cu}(\mathrm{In}, \mathrm{Ga}) \mathrm{Se}_{2} / \mathrm{CdS}$ interfaces using hard $\mathrm{x}$-ray 
photoelectron spectroscopy, Thin Solid Films 582, 366 (2015).

[44] A. O. Pudov, J. R. Sites, M. A. Contreras, T. Nakada, and H.-W. Schock, CIGS $J$-V distortion in the absence of blue photons, Thin Solid Films 480-481, 273 (2005).

[45] A. A. Rockett, D. W. Miller, and J. D. Cohen, Analysis of $\mathrm{CuIn}_{(x)} \mathrm{Ga}_{(1-x)} \mathrm{Se}_{2}$ solar cells using admittance spectroscopy under light bias, in Proceedings of the 38th IEEE Photovoltaic Specialists Conference, Austin, TX, 2012 (IEEE, New York, 2012), p. 1727.

[46] B. Hirschorn, M. E. Orazem, B. Tribollet, V. Vivier, I. Frateur, and M. Musiani, Determination of effective capacitance and film thickness from constant-phase-element parameters, Electrochim. Acta 55, 6218 (2010). 